APS

physics

This is the accepted manuscript made available via CHORUS. The article has been published as:

\title{
Mass measurement of $\wedge\{51\}$ Fe for the determination of the $\wedge\{51\} \mathrm{Fe}(p, \gamma)^{\wedge}\{52\}$ Co reaction rate
}

W.-J. Ong, A. A. Valverde, M. Brodeur, G. Bollen, M. Eibach, K. Gulyuz, A. Hamaker, C. Izzo,

D. Puentes, M. Redshaw, R. Ringle, R. Sandler, S. Schwarz, C. S. Sumithrarachchi, J.

Surbrook, A. C. C. Villari, and I. T. Yandow

Phys. Rev. C 98, 065803 - Published 10 December 2018

DOI: 10.1103/PhysRevC.98.065803 


\title{
Mass Measurement of ${ }^{51} \mathrm{Fe}$ for the Determination of the ${ }^{51} \mathrm{Fe}(\mathrm{p}, \gamma){ }^{52} \mathrm{Co}$ Reaction Rate
}

\author{
W.-J. Ong, ${ }^{1,2}$ A.A. Valverde ${ }^{3}$ M. Brodeur, ${ }^{3}$ G. Bollen, ${ }^{1,4}$ M. Eibach,${ }^{5,2}$ K. Gulyuz, ${ }^{2}$ \\ A. Hamaker,${ }^{1,2}$ C. Izzo, ${ }^{1,2}$ D. Puentes,${ }^{1,2}$ M. Redshaw,${ }^{2,6}$ R. Ringle,${ }^{2}$ R. Sandler, ${ }^{6}$ S. \\ Schwarz, ${ }^{2}$ C.S. Sumithrarachchi, ${ }^{2}$ J. Surbrook, ${ }^{1,2}$ A.C.C. Villari, ${ }^{4}$ and I.T. Yandow ${ }^{1,2}$ \\ ${ }^{1}$ Department of Physics and Astronomy, Michigan State University, East Lansing, Michigan 48824, USA \\ ${ }^{2}$ National Superconducting Cyclotron Laboratory, East Lansing, Michigan 48824 USA \\ ${ }^{3}$ Department of Physics, University of Notre Dame, Notre Dame, Indiana 46556, USA \\ ${ }^{4}$ Facility for Rare Isotope Beams, East Lansing, Michigan 48824 USA \\ ${ }^{5}$ Institut für Physik, Ernst-Moritz-Arndt-Universität, 17487 Greifswald, Germany \\ ${ }^{6}$ Department of Physics, Central Michigan University, Mount Pleasant, Michigan 48859, USA
}

(Dated: November 13, 2018)

\begin{abstract}
Background: The ${ }^{51} \mathrm{Fe}(\mathrm{p}, \gamma){ }^{52} \mathrm{Co}$ reaction lies along the main rp-process path leading up to the ${ }^{56} \mathrm{Ni}$ waiting point. The uncertainty in the reaction $Q$-value, which determines the equilibrium between the forward protoncapture and reverse photo-disintegration ${ }^{52} \mathrm{Co}(\gamma, \mathrm{p}){ }^{51} \mathrm{Fe}$ reaction, contributes to considerable uncertainty in the reaction rate in the temperature range of interest for Type I X-ray bursts and thus to an $\approx 10 \%$ uncertainty in burst ashes lighter than $A=56$.
\end{abstract}

Purpose: With a recent Penning trap mass measurement of ${ }^{52} \mathrm{Co}$ reducing the uncertainty on its mass to 6.6 $\mathrm{keV}$ [J. Phys. G 44, 065103 (2017)], the dominant source of uncertainty in the reaction $Q$-value is now the mass of ${ }^{51} \mathrm{Fe}$, reported in the 2016 atomic mass evaluation to a precision of $9 \mathrm{keV}$ [Chin. Phys. C 41 030003]. A new, high-precision Penning trap mass measurement of ${ }^{51} \mathrm{Fe}$ was performed to allow the determination of an improved precision $Q$-value and thus new reaction rates.

Method: ${ }^{51} \mathrm{Fe}$ was produced using projectile fragmentation at the Coupled Cyclotron Facility at the National Superconducting Cyclotron Laboratory, and separated using the A1900 fragment separator. The resulting secondary beam was then thermalized in the beam stopping area before a mass measurement was performed using the LEBIT 9.4T Penning trap mass spectrometer.

Results: The new mass excess, $\mathrm{ME}=-40189.2(1.6) \mathrm{keV}$, is sixfold more precise than the current AME value, and $1.6 \sigma$ less negative. This value was used to calculate a new proton separation energy for ${ }^{52} \mathrm{Co}$ of $1431(7) \mathrm{keV}$. New excitation levels were then calculated for ${ }^{52} \mathrm{Co}$ using the NUSHELLX code with the GXPF1A interaction, and a new reaction rate and burst ash composition was calculated.

Conclusions: With a new measured $Q$-value, the uncertainty on the ${ }^{51} \mathrm{Fe}(\mathrm{p}, \gamma)$ reaction rate is dominated by the poorly measured ${ }^{52} \mathrm{Co}$ level structure. Reducing this uncertainty would allow a more precise rate calculation and a better determination of the mass abundances in the burst ashes.

\section{INTRODUCTION}

Type I X-ray bursts occur due to explosive burning of $\mathrm{H} / \mathrm{He}$-rich material accreted onto a neutron star from its companion star [1]. During this event, progressively heavier elements are formed through a sequence of the $3 \alpha$ process, the $\alpha, p$ process, and finally the rapid proton capture or rp-process. In order to accurately understand the physics of X-ray bursts, as well as to glean insights regarding the properties of the host neutron star, the nuclear physics of these processes must be well-known.

The rp-process is a series of proton captures and $\beta$ decays that proceeds close to the proton drip line, where the properties of many of the nuclei are poorly, or completely, unknown. Recent sensitivity studies of reaction rates [2] and masses [3] demonstrate the large magnitude of variation when modeling the X-ray burst light curve and rp-process ash composition that arises due to uncertainties in nuclear quantities. There has also been a concurrent effort to measure the reaction rates and masses of nuclei that have been identified as the largest sources of uncertainty.

The reaction ${ }^{51} \mathrm{Fe}(p, \gamma){ }^{52} \mathrm{Co}$ lies along the main rp- process path leading up to the ${ }^{56} \mathrm{Ni}$ waiting point. The current reaction rates recommended by the commonlyused REACLIB reaction rate database [4] span two orders of magnitude in the temperature range of interest for X-ray bursts $\left(0.1 \lesssim T_{9} \lesssim 3.0\right)$, which leads to $\mathrm{a} \approx 10$ $15 \%$ difference in $\mathrm{A}=51$ burst ashes and up to $\approx 5 \%$ differences in mass 52-56 ashes. Existing uncertainty in the reaction $Q$-value, which determines the conditions under which ${ }^{51} \mathrm{Fe}(p, \gamma){ }^{52} \mathrm{Co}$ comes into equilibrium with the photo-disintegration reverse reaction ${ }^{52} \mathrm{Co}(\gamma, p){ }^{51} \mathrm{Fe}$, also leads to a similar order of magnitude uncertainty in the burst ash composition.

To reduce this uncertainty, we report the first Penning trap mass measurement of ${ }^{51} \mathrm{Fe}$. The current mass excess of ${ }^{51} \mathrm{Fe}$ given in the most recent atomic mass evaluation (AME2016 [5]) is $-40203(9) \mathrm{keV}$ and is largely based on the most recent storage ring mass measurement of 40192(11) keV [6]. With the recent JYFLTRAP mass measurement of ${ }^{52} \mathrm{Co}$ [7] that reduced the uncertainty of the ${ }^{52} \mathrm{Co}$ mass excess to several $\mathrm{keV}$, the remaining $Q$-value uncertainty arose from ${ }^{51} \mathrm{Fe}$.

With the new reaction $Q$-value and a new shell model calculation of states in ${ }^{52} \mathrm{Co}$, we recommend new ${ }^{51} \mathrm{Fe}(p, \gamma){ }^{52} \mathrm{Co}$ and ${ }^{52} \mathrm{Co}(\gamma, p){ }^{51} \mathrm{Fe}$ reaction rates, investi- 


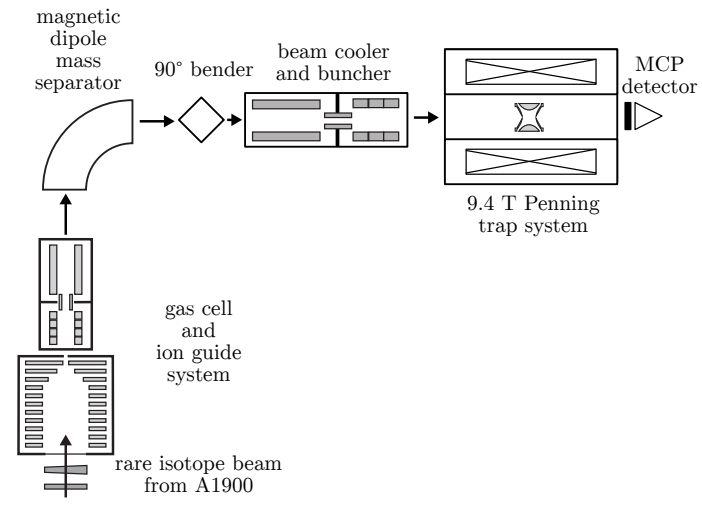

FIG. 1. A schematic diagram showing the major elements of the gas cell and LEBIT facility.

gate the impact of the new rates through single zone $\mathrm{X}$ ray burst simulations, and discuss the remaining sources of uncertainty.

\section{METHOD}

${ }^{51} \mathrm{Fe}$ was produced at the National Superconducting Cyclotron Laboratory (NSCL) and measured at the LowEnergy Beam and Ion Trap (LEBIT) facility [8]. The LEBIT facility is unique among Penning trap mass spectrometry facilities in its ability to perform high-precision mass measurements on rare isotopes produced by projectile fragmentation. A schematic of the gas cell and LEBIT facility at the NSCL can be seen in Fig. 1. In this experiment, radioactive ${ }^{51} \mathrm{Fe}$ was produced by impinging a $160 \mathrm{MeV} / \mathrm{u}$ primary beam of ${ }^{58} \mathrm{Ni}$ on a 752 $\mathrm{mg} / \mathrm{cm}^{2}$ beryllium target at the Coupled Cyclotron Facility at the NSCL. The resulting beam passed through the A1900 fragment separator with a $240 \mathrm{mg} / \mathrm{cm}^{2}$ aluminum wedge $[9]$ to separate the secondary beam. This beam consisted of ${ }^{51} \mathrm{Fe}(11.8 \%)$, with contaminants of ${ }^{50} \mathrm{Mn},{ }^{49} \mathrm{Cr}$, and ${ }^{48} \mathrm{~V}$.

The beam then entered the beam stopping area [10] through a momentum compression beamline, where it was degraded with aluminum degraders of $87 \mathrm{mg} / \mathrm{cm}^{2}$ and $270 \mathrm{mg} / \mathrm{cm}^{2}$ thickness before passing through a 275 $\mathrm{mg} / \mathrm{cm}^{2}, 4.5 \mathrm{mrad}$ aluminum wedge and entering the gas cell with an energy of less than $1 \mathrm{MeV} / \mathrm{u}$. In the gas cell, ions are stopped through collisions with the highpurity helium gas at a pressure of about 73 mbar; during this process, the highly-charged ions recombine down to a singly charged state. These ions were transported by a combination of RF and DC fields as well as gas flow through the gas cell, and were then extracted into a radiofrequency quadrupole (RFQ) ion-guide and transported through a magnetic dipole mass separator with a resolving power greater than 500. Transmitted activity after the mass filter was measured using an insertable $\mathrm{Si}$ detector, and confirmed to be present at $A / Q=51$.

In the LEBIT facility, the ${ }^{51} \mathrm{Fe}^{+}$ions first entered the
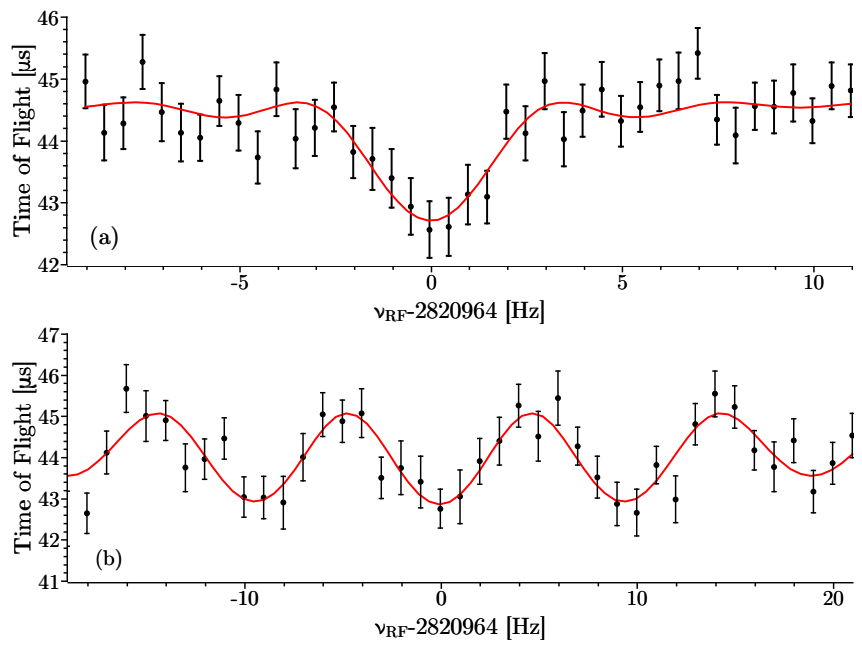

FIG. 2. (color online). A sample $250-\mathrm{ms}^{51} \mathrm{Fe}^{+}$time-offlight traditional continuous cyclotron resonance (top) and $125-\mathrm{ms}^{51} \mathrm{Fe}^{+}$time-of-flight Ramsey cyclotron resonance (bottom) used for the determination of the frequency ratio of $\nu_{\text {ref }}^{\text {int }}\left(\mathrm{CH}_{2}{ }^{37} \mathrm{Cl}^{+}\right) / \nu_{c}\left({ }^{51} \mathrm{Fe}^{+}\right)$. The solid red curves represents a fit of the theoretical profile $[16,17]$.

cooler-buncher, a multi-staged helium-gas-filled RFQ ion trap [11]. In the first stage, moderate pressure $\left(\approx 10^{-3}\right.$ mbar) helium gas was used to cool the ions in a large diameter RFQ ion guide. In the final, ions were accumulated, cooled, and released to the LEBIT Penning trap in pulses of approximately $100 \mathrm{~ns}$ [12]. A fast kicker in the beam line between the cooler-buncher and the Penning trap was used as a time-of-flight mass separator to further purify the beam, selecting ions of $A / Q=51$, corresponding to ${ }^{51} \mathrm{Fe}^{+}$and contaminants of the same $A / Q$.

The 9.4T Penning trap at the LEBIT facility consists of a high-precision hyperbolic electrode system contained in an actively-shielded $9.4 \mathrm{~T}$ magnet system [8]. Electrodes in front of the Penning trap are used to decelerate the ion pulses to low energy before entering the trap. The final section of these electrodes are quadrisected radially to form a "Lorentz steerer" [13] that forces the ions to enter the trap off-axis and perform a magnetron motion of frequency $\nu_{-}$once the trapping potential is switched on.

After their capture, the trapped ions were purified, using the dipole cleaning technique [14], reducing the abundance of contaminants to less than a few percent. In this technique, azimuthal RF dipole fields are used to excite contaminant ions at their specific reduced cyclotron frequency $\nu_{+}$and thus drive them to a large enough radius such that they do not interfere with the measurement. Then, the time-of-flight cyclotron resonance technique (TOF-ICR) $[15,16]$ was used to determine the ions' cyclotron frequency. From this resonance one can measure the cyclotron frequency $\nu_{c}=q B /(2 \pi m)$ and so determine the mass $m$ for a known charge $q$ and magnetic field strength $B$.

In this measurement, 50 and $250 \mathrm{~ms}$ continuous 
quadrupole resonances and 125 ms Ramsey quadrupole resonances were used. In a continuous resonance, either 50 or $250 \mathrm{~ms}$ of continuous RF quadrupole excitation are applied in a single pulse [16]; for a Ramsey resonance, a two-pulse excitation scheme was used, where two 25 ms quadrupole RF pulses were separated by a $75 \mathrm{~ms}$ waiting time [17-19]. Afterwards, a fit to the theoretical line shape was performed to determine the cyclotron frequency; sample fits of both types can be seen in Fig. 2. Between measurements of ${ }^{51} \mathrm{Fe}^{+}$, measurements of the cyclotron frequency of the reference chloromethyl ion, ${ }^{12} \mathrm{C}^{1} \mathrm{H}_{2}{ }^{37} \mathrm{Cl}^{+}$, were taken. The chloromethyl was produced in the gas cell.

\section{RESULTS}

\section{A. New mass excess of ${ }^{51} \mathrm{Fe}$}

In Penning trap mass spectrometry, the experimental result is the ratio $R=\nu_{\text {ref }}^{\text {int }}\left(\mathrm{CH}_{2} \mathrm{Cl}^{+}\right) / \nu_{c}\left({ }^{51} \mathrm{Fe}^{+}\right)$, where in this case, $\nu_{\text {ref }}^{\text {int }}$ is the interpolated frequency of the chloromethyl reference ion bracketing the measurement of $\nu_{c}$, the cyclotron frequency of ${ }^{51} \mathrm{Fe}^{+}$. A series of five measurements was taken over the course of four hours, resulting in a weighted average of $\bar{R}=0.999515555(29)$. These measurements are shown in Fig. 3. The Birge ratio [20] of $0.54(21)$, less than one, indicates that the statistical uncertainties on the individual measurements has not been underestimated.

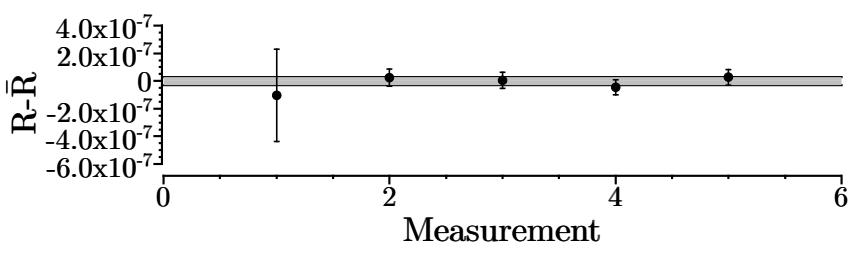

FIG. 3. Measured cyclotron frequency ratios $R=$ $\nu_{\text {ref }}^{\text {int }}\left(\mathrm{CH}_{2}{ }^{37} \mathrm{Cl}\right) / \nu_{c}\left({ }^{51} \mathrm{Fe}\right)$ relative to the weighted average $\bar{R}$. The grey bar represents the $1 \sigma$ uncertainty in $\bar{R}$.

Previous work has shown that the effect of nonlinear magnetic field fluctuations on the ratio $R$ is less than $1 \times 10^{-9}$ per hour [21], longer than our measurement time. The presence of isobaric contaminants in the trap during a measurement could also lead to a systematic frequency shift [22]. This effect was minimized by ensuring no contaminants were present at a level exceeding a few percent through cleaning and by limiting the total number of ions in the trap. This was done by analyzing events with five or fewer detected ions, corresponding to eight or fewer ions in the trap based on our measured MCP efficiency of $63 \%$ [23]. Furthermore, a $z$-class analysis was performed, and any count-dependent systematic shifts were found to be more than an order of magnitude smaller than the statistical uncertainty. $\mathrm{As}^{51} \mathrm{Fe}^{+}$and the chloromethyl ion form an isobaric doublet, most of the mass-dependent systematic shifts, such as relativistic shifts due to differences in velocity and in orbital radii and shifts due to trap field imperfections, are eliminated; previous work at LEBIT has shown these shifts to be $\Delta R=2 \times 10^{-10} / \mathrm{u}[24]$, so for sub-u differences, such shifts are negligible compared to the statistical uncertainty.

The ${ }^{51} \mathrm{Fe}$ mass was then calculated following:

$$
M\left({ }^{51} \mathrm{Fe}\right)=\bar{R}\left[M\left({ }^{12} \mathrm{C}^{1} \mathrm{H}_{2}{ }^{37} \mathrm{Cl}\right)-m_{e}\right]+m_{e},
$$

where $m_{e}$ the electron mass and $M\left({ }^{12} \mathrm{C}^{1} \mathrm{H}_{2}{ }^{37} \mathrm{Cl}\right)$ is the mass of the chloromethyl ion, calculated from AME2016. The electron ionization energies of iron and chloromethyl and the molecular binding energy of choloromethyl ion are both on the order of eVs and thus were not included as they are several orders of magnitude smaller than the statistical uncertainty of the measurement. The calculated mass excess of ${ }^{51} \mathrm{Fe}$ is $M E=-40189.2(1.6)$ $\mathrm{keV}$, which is over sixfold more precise than the current AmE2016 value, $M E=-40203(9) \mathrm{keV}$, and $1.6 \sigma$ less negative [5]. With this new value, the proton separation energy of ${ }^{52} \mathrm{Co}$, calculated with the newest ${ }^{52} \mathrm{Co}$ mass from [7], is $1431(7) \mathrm{keV}$.

\section{B. ${ }^{51} \mathrm{Fe}(p, \gamma)^{52} \mathrm{Co}$ reaction rate}

The ${ }^{51} \mathrm{Fe}(p, \gamma){ }^{52} \mathrm{Co}$ reaction rate remains uncertain. Constraints on the direct capture and resonant capture rates are weak as only a few excited states in ${ }^{52} \mathrm{Co}$ have been identified by experimental data [25]. The direct capture component does not contribute significantly at the relevant temperatures, as found in [26]. The resonant capture component that dominates the total reaction rate (in REACLIB) is currently purely based on a shell model calculation by [26], which used a modified KB3 interaction in the $p f$-shell [27]. To update the reaction rate, taking into account the new reaction $Q$-value, a shell model calculation using the code NUSHELLX [28] was performed. The calculation allowed up to 3 particle3 hole excitations in the $p f$-shell on top of a closed ${ }^{40} \mathrm{Ca}$ core, using the newer GXPF1A interaction [29]. The results of the calculation, including spectroscopic factors $C^{2} S$, proton widths $\Gamma_{p}$, and $\gamma$ widths $\Gamma_{\gamma}$, are listed in Tab. I.

A Monte Carlo approach [30, 31] was used to estimate the uncertainty in the reaction rate based on the uncertainty in the shell model excitation energies. Each level was assumed to vary within a Gaussian distribution centered at the calculated value and with a width of $200 \mathrm{keV}$. The resultant distribution of rates for a given $T_{9}$ was sampled to obtain the $16^{\text {th }}, 50^{\text {th }}$, and $84^{\text {th }}$ percentiles, corresponding to the $1 \sigma$ lower, median, and $1 \sigma$ upper reaction rate. This was done for a range of temperatures between 0.1 and 10 GK to obtain the final rate uncertainty. 
TABLE I. New shell model excitation levels for ${ }^{52}$ Co over the proton separation energy and up to $4 \mathrm{MeV}$. Spectroscopic factors $C^{2} S$ used to calculate the partial proton and gamma widths ( $\Gamma_{p}$ and $\Gamma_{\gamma}$ respectively) were calculated utilizing the shell model with the GXPF1A interaction [29] that allowed up to 3 particle- 3 hole configurations. Only states above the proton separation energy are listed.

\begin{tabular}{|c|c|c|c|c|c|c|}
\hline \multicolumn{3}{|c|}{ Levels (keV) } & \multicolumn{2}{|c|}{$C^{2} S$} & \multicolumn{2}{|c|}{$\Gamma(\mathrm{eV})$} \\
\hline$E_{x}$ & $E_{\text {res }}$ & $\mathrm{J}^{\pi}$ & $l=1$ & $l=3$ & $\Gamma_{\gamma}$ & $\Gamma_{p}$ \\
\hline 1560 & 129 & $5^{+}$ & $4.17 \times 10^{-2}$ & $4.17 \times 10^{-2}$ & $8.26 \times 10^{-4}$ & $4.12 \times 10^{-20}$ \\
\hline 1601 & 170 & $4^{+}$ & $2.94 \times 10^{-1}$ & $2.94 \times 10^{-1}$ & $4.21 \times 10^{-4}$ & $1.32 \times 10^{-15}$ \\
\hline 1716 & 285 & $4^{+}$ & $2.28 \times 10^{-1}$ & $2.46 \times 10^{-2}$ & $1.57 \times 10^{-4}$ & $3.67 \times 10^{-9}$ \\
\hline 1818 & 387 & $6^{+}$ & & $3.38 \times 10^{-2}$ & $1.84 \times 10^{-2}$ & $1.57 \times 10^{-9}$ \\
\hline 1954 & 523 & $5^{+}$ & & $1.12 \times 10^{-1}$ & $1.53 \times 10^{-2}$ & $4.40 \times 10^{-7}$ \\
\hline 2027 & 596 & $4^{+}$ & $1.03 \times 10^{-2}$ & $4.26 \times 10^{-2}$ & $1.65 \times 10^{-2}$ & $2.89 \times 10^{-5}$ \\
\hline 2047 & 616 & $3^{+}$ & $9.10 \times 10^{-3}$ & $1.02 \times 10^{-1}$ & $7.18 \times 10^{-3}$ & $3.93 \times 10^{-5}$ \\
\hline 2126 & 695 & $5^{+}$ & & $4.49 \times 10^{-2}$ & $8.58 \times 10^{-3}$ & $6.08 \times 10^{-6}$ \\
\hline 2161 & 730 & $2^{+}$ & $8.59 \times 10^{-2}$ & $2.10 \times 10^{-1}$ & $2.00 \times 10^{-2}$ & $3.02 \times 10^{-3}$ \\
\hline 2262 & 831 & $3^{+}$ & $5.00 \times 10^{-4}$ & $1.11 \times 10^{-1}$ & $4.25 \times 10^{-3}$ & $7.65 \times 10^{-5}$ \\
\hline 2263 & 832 & $5^{+}$ & & $2.25 \times 10^{-2}$ & $6.90 \times 10^{-3}$ & $2.14 \times 10^{-5}$ \\
\hline 2358 & 927 & $1^{+}$ & $2.00 \times 10^{-3}$ & $1.27 \times 10^{-2}$ & $2.67 \times 10^{-2}$ & $9.74 \times 10^{-4}$ \\
\hline 2415 & 984 & $6^{+}$ & & $5.20 \times 10^{-3}$ & $3.55 \times 10^{-4}$ & $2.55 \times 10^{-5}$ \\
\hline 2498 & 1067 & $1^{+}$ & $2.10 \times 10^{-3}$ & $3.00 \times 10^{-4}$ & $1.81 \times 10^{-3}$ & $4.09 \times 10^{-3}$ \\
\hline 2521 & 1090 & $5^{+}$ & & $2.00 \times 10^{-4}$ & $5.03 \times 10^{-3}$ & $2.47 \times 10^{-6}$ \\
\hline 2575 & 1144 & $2^{+}$ & $3.79 \times 10^{-2}$ & $2.08 \times 10^{-2}$ & $1.51 \times 10^{-2}$ & $1.41 \times 10^{-1}$ \\
\hline 2603 & 1172 & $4^{+}$ & $7.70 \times 10^{-3}$ & $3.10 \times 10^{-2}$ & $2.79 \times 10^{-3}$ & $3.56 \times 10^{-2}$ \\
\hline 2641 & 1210 & $3^{+}$ & $2.42 \times 10^{-2}$ & $8.10 \times 10^{-2}$ & $8.16 \times 10^{-3}$ & $1.48 \times 10^{-1}$ \\
\hline 2649 & 1218 & $6^{+}$ & & $2.50 \times 10^{-3}$ & $3.00 \times 10^{-3}$ & $7.91 \times 10^{-5}$ \\
\hline 2667 & 1236 & $1^{+}$ & $4.02 \times 10^{-1}$ & $3.47 \times 10^{-2}$ & $1.28 \times 10^{-2}$ & 2.97 \\
\hline 2701 & 1270 & $4^{+}$ & $1.03 \times 10^{-2}$ & $2.30 \times 10^{-1}$ & $1.78 \times 10^{-2}$ & $9.61 \times 10^{-2}$ \\
\hline 2716 & 1285 & $2^{+}$ & $6.55 \times 10^{-2}$ & $1.66 \times 10^{-2}$ & $1.16 \times 10^{-2}$ & $6.75 \times 10^{-1}$ \\
\hline 2776 & 1345 & $2^{+}$ & $1.43 \times 10^{-2}$ & $1.50 \times 10^{-3}$ & $8.05 \times 10^{-3}$ & $2.16 \times 10^{-1}$ \\
\hline 2801 & 1370 & $3^{+}$ & $4.41 \times 10^{-2}$ & $2.34 \times 10^{-2}$ & $2.61 \times 10^{-2}$ & $7.75 \times 10^{-1}$ \\
\hline 2839 & 1408 & $3^{+}$ & $4.00 \times 10^{-4}$ & $8.10 \times 10^{-3}$ & $5.30 \times 10^{-3}$ & $8.77 \times 10^{-3}$ \\
\hline 2879 & 1448 & $0^{+}$ & & $6.00 \times 10^{-4}$ & $5.00 \times 10^{-4}$ & $1.65 \times 10^{-2}$ \\
\hline 2919 & 1488 & $6^{+}$ & & $1.80 \times 10^{-3}$ & $1.23 \times 10^{-2}$ & $2.70 \times 10^{-4}$ \\
\hline 2920 & 1489 & $3^{+}$ & $3.30 \times 10^{-3}$ & $4.00 \times 10^{-3}$ & $2.37 \times 10^{-3}$ & $1.13 \times 10^{-1}$ \\
\hline 2921 & 1490 & $0^{+}$ & & $8.90 \times 10^{-3}$ & $7.64 \times 10^{-2}$ & $1.34 \times 10^{-3}$ \\
\hline 2943 & 1512 & $1^{+}$ & $6.82 \times 10^{-2}$ & $6.70 \times 10^{-3}$ & $5.62 \times 10^{-2}$ & 2.62 \\
\hline 2954 & 1523 & $4^{+}$ & $4.20 \times 10^{-3}$ & $5.90 \times 10^{-3}$ & $6.70 \times 10^{-3}$ & $1.71 \times 10^{-1}$ \\
\hline 2992 & 1561 & $6^{+}$ & & $5.00 \times 10^{-4}$ & $1.07 \times 10^{-2}$ & $1.05 \times 10^{-4}$ \\
\hline
\end{tabular}

The new reaction rate, calculated with the new $Q$-value taking into account the latest JYFLTRAP ${ }^{52}$ Co mass result and the ${ }^{51} \mathrm{Fe}$ mass reported here, is listed for certain temperatures between 0.1-10 GK in Tab. II, and in the REACLIB format in Tab. III. A comparison with the previous reaction rate (which used a $Q$-value of $986 \mathrm{keV}$ from AME2003 [32]) is shown in Fig. 4. The impact of the higher $Q$-value is apparent in Fig. 4, where the intersection of the forward and reverse reaction rates is $\approx 0.2$ GK higher when compared to the current REACLIB rate. Because the ${ }^{51} \mathrm{Fe}(p, \gamma)$ rate is occurring close to peak temperatures of the X-ray burst, the reaction flow is particularly sensitive to where this intersection lies. The result of the higher ratio is shown in Fig. 5, where the new shell model rate (even within its 1- $\sigma$ error bar) results in a faster reaction flow to higher masses, depleting the final $\mathrm{A}=51$ abundance and enhancing the $\mathrm{A}=$ 52-55 abundances. The enhancement in this mass region is especially important given that $\mathrm{A}=53-65$ nuclei are some of the biggest contributors to Urca cooling in the neutron star crust during the quiescent phase.

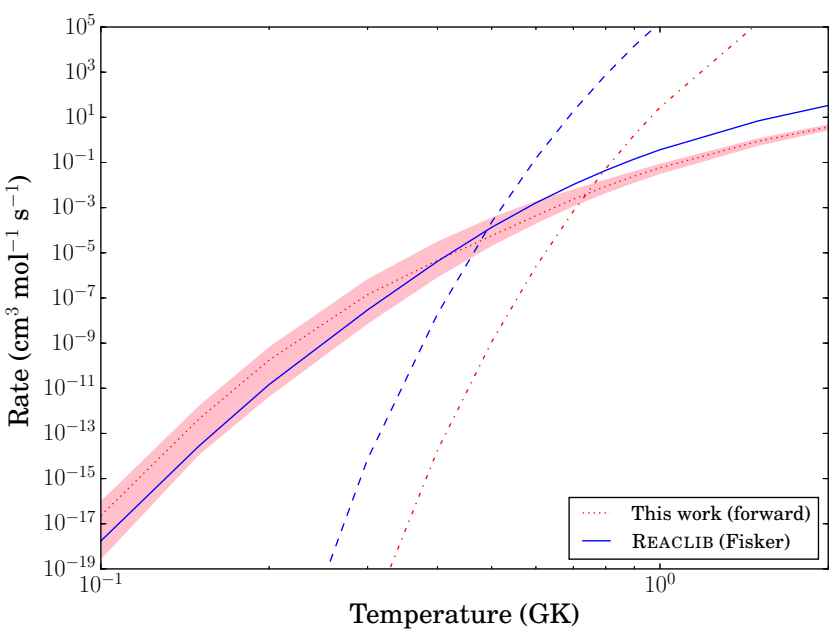

FIG. 4. Color online: Forward and reverse rates for the current recommended rate in REACLIB (blue solid and dashed respectively) and the new recommended rate (red dotted and dot-dashed respectively). The pale red band represents the current $1-\sigma$ uncertainty range in the reaction rate.

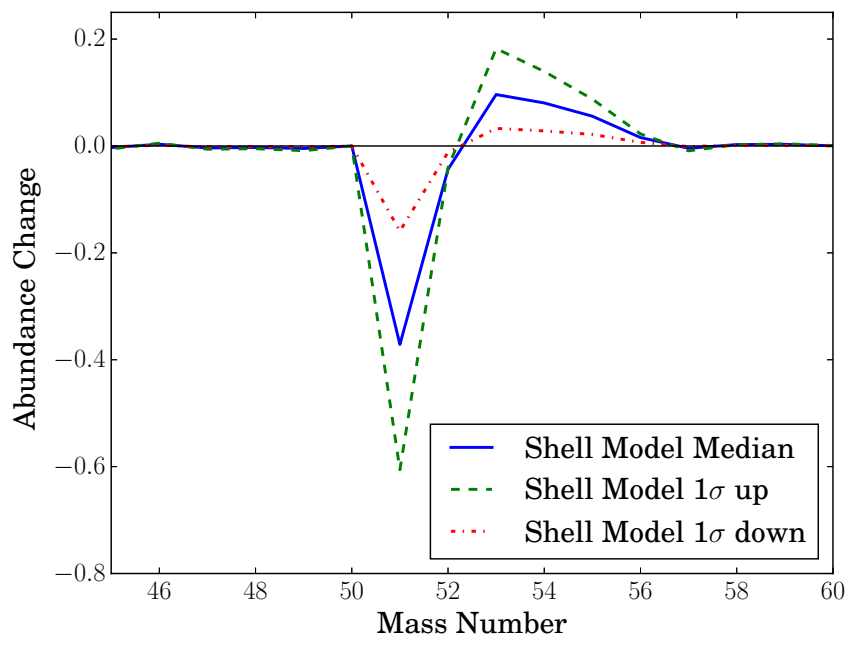

FIG. 5. Color online: Fractional differences in abundance relative to the current REACLIB rate for the median (blue solid), $1 \sigma$ up (green dashed), and $1 \sigma$ down (red dot-dashed) of the new reaction rate distribution. 


\section{CONCLUSIONS}

The first Penning trap mass measurement of ${ }^{51} \mathrm{Fe}$ was completed, producing a value with a precision of $1.6 \mathrm{keV}$, a sixfold improvement over the current AME value, which along with the recent Penning trap mass measurement of ${ }^{52} \mathrm{Co}$ allowed the calculation of the ${ }^{52} \mathrm{Co}$ proton separation to a precision of $7 \mathrm{keV}$, improving on the precision of the previous value and shifting it down by $1.3 \sigma$. Single-zone calculations incorporating the new $Q$-value show that uncertainties of several orders of magnitude in the ${ }^{51} \mathrm{Fe}(p, \gamma)$ reaction rate lead to $\approx 10 \%$ variations in the abundances of mass 51-55 nuclei, which are some of the most abundant nuclei produced in typical X-ray bursts. However, the unknown ${ }^{52}$ Co level scheme and the resultant large uncertainty in the ${ }^{51} \mathrm{Fe}(p, \gamma)$ reaction rate still contributes significantly to the uncertainty in the ash composition. Thus, an experimental determination of the ${ }^{52} \mathrm{Co}$ level scheme is crucial for more precise calculations of X-ray burst ashes.

Finally, constraints on the composition of X-ray burst ashes are important for observations of the cooling neutron star when it enters its quiescent phase [33]. Previous studies modeling neutron star cooling curves show that there are degeneracies between different physical properties in terms of their impact on the thermal evolution of the neutron star [34]. The impurity parameter $Q$ that measures the inhomogeneity of the outer layers of the neutron star crust is one of the most important of these properties, and is a direct consequence of the rp-process ashes that formed the new crust. Constraints on $Q$ would help to break these degeneracies, and provide more information about the neutron star properties, potentially including the origin of the postulated shallow heat source.

\section{ACKNOWLEDGMENTS}

The authors would like to thank Hendrik Schatz for helpful discussions regarding the rp-process calculations. This work was conducted with the support of Michigan State University, the National Science Foundation under Grants No. PHY-1102511, No. PHY-1713857 and No. PHY-1430152 (JINA Center for the Evolution of the Elements), and the US Department of Energy, Office of Science, Office of Nuclear Physics under award number DESC0015927. The work leading to this publication has also been supported by a DAAD P.R.I.M.E. fellowship with funding from the German Federal Ministry of Education and Research and the People Programme (Marie Curie Actions) of the European Union's Seventh Framework Programme FP7/2007/2013) under REA Grant Agreement No. 605728.
[1] S. E. Woosley and R. E. Taam, Nature (London) 263, 101 (1976).

[2] R. H. Cyburt, A. M. Amthor, A. Heger, E. Johnson, L. Keek, Z. Meisel, H. Schatz, and K. Smith, The Astrophysical Journal 830, 55 (2016).

[3] H. Schatz and W.-J. Ong, Astrophys. J. 844, 139 (2017), arXiv:1610.07596 [astro-ph.HE].

[4] R. H. Cyburt, A. M. Amthor, R. Ferguson, Z. Meisel, K. Smith, S. Warren, A. Heger, R. D. Hoffman, T. Rauscher, A. Sakharuk, H. Schatz, F. K. Thielemann, and M. Wiescher, ApJ 189, 240 (2010).

[5] M. Wang, G. Audi, F. Kondev, W. Huang, S. Naimi, and X. Xu, Chinese Physics C 41, 030003 (2017).

[6] X. Tu, M. Wang, Y. Litvinov, Y. Zhang, H. Xu, Z. Sun, G. Audi, K. Blaum, C. Du, W. Huang, Z. Hu, P. Geng, S. Jin, L. Liu, Y. Liu, B. Mei, R. Mao, X. Ma, H. Suzuki, P. Shuai, Y. Sun, S. Tang, J. Wang, S. Wang, G. Xiao, X. Xu, J. Xia, J. Yang, R. Ye, T. Yamaguchi, X. Yan, Y. Yuan, Y. Yamaguchi, Y. Zang, H. Zhao, T. Zhao, X. Zhang, X. Zhou, and W. Zhan, Nuclear Instruments and Methods in Physics Research Section A: Accelerators, Spectrometers, Detectors and Associated Equipment 654, 213 (2011).

[7] D. A. Nesterenko, A. Kankainen, L. Canete, M. Block, D. Cox, T. Eronen, C. Fahlander, U. Forsberg, J. Gerl, P. Golubev, J. Hakala, A. Jokinen, V. S. Kolhinen, J. Koponen, N. Lalović, C. Lorenz, I. D. Moore, P. Papadakis, J. Reinikainen, S. Rinta-Antila, D. Rudolph, L. G. Sarmiento, A. Voss, and J. Äystö, Journal of Physics G Nuclear Physics 44, 065103 (2017), arXiv:1701.04069 [nucl-ex].

[8] R. Ringle, G. Bollen, and S. Schwarz, Int. J. Mass Spectrom. 349-350, 87 (2013).

[9] D. J. Morrissey, B. M. Sherrill, M. Steiner, A. Stolz, and I. Wiedenhoever, Nucl. Instrum. Methods Phys. Res., Sect. B 204, 90 (2003).

[10] K. Cooper, C. S. Sumithrarachchi, D. J. Morrissey, A. Levand, J. A. Rodriguez, G. Savard, S. Schwarz, and B. Zabransky, Nucl. Instrum. Methods Phys. Res., Sect. A 763, 543 (2014).

[11] S. Schwarz, G. Bollen, D. Lawton, A. Neudert, R. Ringle, P. Schury, and T. Sun, Nucl. Instrum. Methods Phys. Res., Sect. B 204, 474 (2003).

[12] R. Ringle, G. Bollen, A. Prinke, J. Savory, P. Schury, S. Schwarz, and T. Sun, Nucl. Instrum. Methods Phys. Res., Sect. A 604, 536 (2009).

[13] R. Ringle, G. Bollen, A. Prinke, J. Savory, P. Schury, S. Schwarz, and T. Sun, Int. J. Mass Spectrom. 263, 38 (2007).

[14] K. Blaum, D. Beck, G. Bollen, P. Delahaye, C. Guénaut, F. Herfurth, A. Kellerbauer, H.-J. Kluge, D. Lunney, S. Schwarz, L. Schweikhard, and C. Yazidjian, EPL (Europhysics Letters) 67, 586 (2004).

[15] G. Bollen, R. B. Moore, G. Savard, and H. Stolzenberg, J. Appl. Phys. 68, 4355 (1990).

[16] M. König, G. Bollen, H.-J. Kluge, T. Otto, and J. Szerypo, Int. J. Mass Spectrom. 142, 95 (1995).

[17] M. Kretzschmar, Int. J. Mass Spectrom. 264, 122 (2007).

[18] G. Bollen, H.-J. Kluge, T. Otto, G. Savard, and H. Stolzenberg, Nucl. Instrum. Methods Phys. Res., Sect. 
B 70, 490 (1992).

[19] S. George, K. Blaum, F. Herfurth, A. Herlert, M. Kretzschmar, S. Nagy, S. Schwarz, L. Schweikhard, and C. Yazidjian, Int. J. Mass Spectrom. 264, 110 (2007).

[20] R. T. Birge, Phys. Rev. 40, 207 (1932).

[21] R. Ringle, T. Sun, G. Bollen, D. Davies, M. Facina, J. Huikari, E. Kwan, D. J. Morrissey, A. Prinke, J. Savory, P. Schury, S. Schwarz, and C. S. Sumithrarachchi, Phys. Rev. C 75, 055503 (2007).

[22] G. Bollen, H.-J. Kluge, M. König, T. Otto, G. Savard, H. Stolzenberg, R. B. Moore, G. Rouleau, G. Audi, and I. Collaboration, Phys. Rev. C.. 46, R2140 (1992).

[23] A. A. Valverde, G. Bollen, M. Brodeur, R. A. Bryce, K. Cooper, M. Eibach, K. Gulyuz, C. Izzo, D. J. Morrissey, M. Redshaw, R. Ringle, R. Sandler, S. Schwarz, C. S. Sumithrarachchi, and A. C. C. Villari, Phys. Rev. Lett. 114, 232502 (2015).

[24] K. Gulyuz, J. Ariche, G. Bollen, S. Bustabad, M. Eibach, C. Izzo, S. J. Novario, M. Redshaw, R. Ringle, R. Sandler, S. Schwarz, and A. A. Valverde, Phys. Rev. C 91, 055501 (2015).

[25] S. E. A. Orrigo, B. Rubio, Y. Fujita, W. Gelletly, J. Agramunt, A. Algora, P. Ascher, B. Bilgier, B. Blank, L. Cáceres, R. B. Cakirli, E. Ganioğlu, M. Gerbaux, J. Giovinazzo, S. Grévy, O. Kamalou, H. C. Kozer, L. Kucuk, T. Kurtukian-Nieto, F. Molina, L. Popescu, A. M. Rogers, G. Susoy, C. Stodel, T. Suzuki, A. Tamii, and J. C. Thomas, Phys. Rev. C 93, 044336 (2016).

[26] J. FISKER, V. BARNARD, J. GÖRRES, K. LANGANKE, G. MARTÍNEZ-PINEDO, and M. WIESCHER, Atomic Data and Nuclear Data Tables 79, 241
$(2001)$

[27] T. T. S. Kuo and G. E. Brown, Nucl. Phys. A114, 241 (1968).

[28] B. A. Brown and W. D. M. Rae, Nuclear Data Sheets 120, 115 (2014).

[29] M. Honma, T. Otsuka, B. A. Brown, and T. Mizusaki, The European Physical Journal A - Hadrons and Nuclei 25 (2005), 10.1140/epjad/i2005-06-032-2.

[30] W.-J. Ong, C. Langer, F. Montes, A. Aprahamian, D. W. Bardayan, D. Bazin, B. A. Brown, J. Browne, H. Crawford, R. Cyburt, E. B. Deleeuw, C. Domingo-Pardo, A. Gade, S. George, P. Hosmer, L. Keek, A. Kontos, I.-Y. Lee, A. Lemasson, E. Lunderberg, Y. Maeda, M. Matos, Z. Meisel, S. Noji, F. M. Nunes, A. Nystrom, G. Perdikakis, J. Pereira, S. J. Quinn, F. Recchia, H. Schatz, M. Scott, K. Siegl, A. Simon, M. Smith, A. Spyrou, J. Stevens, S. R. Stroberg, D. Weisshaar, J. Wheeler, K. Wimmer, and R. G. T. Zegers, Phys. Rev. C 95, 055806 (2017).

[31] C. Iliadis, R. Longland, A. Coc, F. X. Timmes, and A. E. Champagne, Journal of Physics G: Nuclear and Particle Physics 42, 034007 (2015).

[32] G. Audi, A. Wapstra, and C. Thibault, Nuclear Physics A 729, 337 (2003), the 2003 NUBASE and Atomic Mass Evaluations.

[33] H. Schatz, S. Gupta, P. Möller, M. Beard, E. F. Brown, A. T. Deibel, L. R. Gasques, W. R. Hix, L. Keek, R. Lau, A. W. Steiner, and M. Wiescher, Nature (London) 505, 62 (2014), arXiv:1312.2513 [astro-ph.SR].

[34] Z. Meisel and A. Deibel, Astrophys. J. 837, 73 (2017), arXiv:1701.02730 [astro-ph.HE]. 
TABLE I. (continued)

\begin{tabular}{|c|c|c|c|c|c|c|}
\hline \multicolumn{3}{|c|}{ Levels (keV) } & \multicolumn{2}{|c|}{$C^{2} S$} & \multicolumn{2}{|c|}{$\Gamma(\mathrm{eV})$} \\
\hline$E_{x}$ & $E_{r e s}$ & $\mathrm{~J}^{\pi}$ & $l=1$ & $l=3$ & $\Gamma_{\gamma}$ & $\Gamma_{p}$ \\
\hline 3020 & 1589 & $2^{+}$ & $1.58 \times 10^{-1}$ & $3.10 \times 10^{-3}$ & $1.37 \times 10^{-2}$ & 8.85 \\
\hline 3021 & 1590 & $5^{+}$ & & $1.10 \times 10^{-3}$ & $3.20 \times 10^{-2}$ & $2.63 \times 10^{-3}$ \\
\hline 3032 & 1601 & $3^{+}$ & $6.34 \times 10^{-2}$ & $1.98 \times 10^{-2}$ & $4.22 \times 10^{-3}$ & 3.76 \\
\hline 3082 & 1651 & $2^{+}$ & $1.78 \times 10^{-2}$ & $1.39 \times 10^{-2}$ & $3.25 \times 10^{-2}$ & 1.33 \\
\hline 3119 & 1688 & $4^{+}$ & $2.47 \times 10^{-2}$ & $1.80 \times 10^{-3}$ & $2.31 \times 10^{-2}$ & 2.16 \\
\hline 3171 & 1740 & $1^{+}$ & $3.74 \times 10^{-2}$ & $2.00 \times 10^{-4}$ & $6.78 \times 10^{-4}$ & 4.07 \\
\hline 3172 & 1741 & $5^{+}$ & & $3.14 \times 10^{-2}$ & $1.27 \times 10^{-2}$ & $1.39 \times 10^{-2}$ \\
\hline 3191 & 1760 & $3^{+}$ & $4.30 \times 10^{-3}$ & $8.50 \times 10^{-3}$ & $1.22 \times 10^{-2}$ & $5.07 \times 10^{-1}$ \\
\hline 3236 & 1805 & $2^{+}$ & $1.29 \times 10^{-1}$ & $1.00 \times 10^{-4}$ & $7.51 \times 10^{-2}$ & $1.82 \times 10^{1}$ \\
\hline 3279 & 1848 & $4^{+}$ & $6.10 \times 10^{-3}$ & $1.06 \times 10^{-2}$ & $8.71 \times 10^{-3}$ & 1.01 \\
\hline 3287 & 1856 & $1^{+}$ & $9.01 \times 10^{-2}$ & $1.92 \times 10^{-2}$ & $7.01 \times 10^{-2}$ & $1.54 \times 10^{1}$ \\
\hline 3289 & 1858 & $5^{+}$ & & $1.10 \times 10^{-3}$ & $1.06 \times 10^{-2}$ & $7.46 \times 10^{-4}$ \\
\hline 3331 & 1900 & $3^{+}$ & $1.60 \times 10^{-3}$ & $2.80 \times 10^{-3}$ & $1.12 \times 10^{-2}$ & $3.20 \times 10^{-1}$ \\
\hline 3333 & 1902 & $2^{+}$ & $8.50 \times 10^{-3}$ & $5.80 \times 10^{-3}$ & $1.60 \times 10^{-3}$ & 1.71 \\
\hline 3398 & 1967 & $4^{+}$ & $1.11 \times 10^{-2}$ & $1.26 \times 10^{-2}$ & $9.06 \times 10^{-3}$ & 2.80 \\
\hline 3413 & 1982 & $1^{+}$ & $1.17 \times 10^{-2}$ & $5.90 \times 10^{-3}$ & $1.99 \times 10^{-3}$ & 3.10 \\
\hline 3427 & 1996 & $2^{+}$ & $3.76 \times 10^{-2}$ & $4.50 \times 10^{-3}$ & $1.14 \times 10^{-2}$ & $1.04 \times 10^{1}$ \\
\hline 3465 & 2034 & $2^{+}$ & $4.00 \times 10^{-4}$ & $1.30 \times 10^{-3}$ & $6.18 \times 10^{-3}$ & $1.25 \times 10^{-1}$ \\
\hline 3503 & 2072 & $5^{+}$ & & $4.00 \times 10^{-4}$ & $1.06 \times 10^{-2}$ & $5.32 \times 10^{-4}$ \\
\hline 3509 & 2078 & $3^{+}$ & $1.64 \times 10^{-2}$ & $4.00 \times 10^{-4}$ & $2.51 \times 10^{-2}$ & 5.88 \\
\hline 3537 & 2106 & $1^{+}$ & $3.52 \times 10^{-2}$ & $2.19 \times 10^{-2}$ & $1.18 \times 10^{-2}$ & $1.38 \times 10^{1}$ \\
\hline 3551 & 2120 & $3^{+}$ & $3.00 \times 10^{-3}$ & $1.00 \times 10^{-3}$ & $8.66 \times 10^{-3}$ & 1.23 \\
\hline 3559 & 2128 & $6^{+}$ & & $1.40 \times 10^{-3}$ & $1.52 \times 10^{-2}$ & $2.19 \times 10^{-3}$ \\
\hline 3575 & 2144 & $5^{+}$ & & $2.30 \times 10^{-2}$ & $8.36 \times 10^{-3}$ & $3.76 \times 10^{-2}$ \\
\hline 3576 & 2145 & $4^{+}$ & $1.46 \times 10^{-2}$ & $5.90 \times 10^{-3}$ & $4.49 \times 10^{-2}$ & 6.42 \\
\hline 3638 & 2207 & $5^{+}$ & & $2.00 \times 10^{-3}$ & $5.00 \times 10^{-3}$ & $3.87 \times 10^{-3}$ \\
\hline 3645 & 2214 & $4^{+}$ & $4.80 \times 10^{-2}$ & $3.49 \times 10^{-2}$ & $1.47 \times 10^{-2}$ & $2.57 \times 10^{1}$ \\
\hline 3654 & 2223 & $1^{+}$ & $4.00 \times 10^{-4}$ & $6.07 \times 10^{-2}$ & $1.28 \times 10^{-2}$ & $2.19 \times 10^{-1}$ \\
\hline 3701 & 2270 & $6^{+}$ & & $6.00 \times 10^{-4}$ & $1.93 \times 10^{-2}$ & $1.36 \times 10^{-3}$ \\
\hline 3708 & 2277 & $0^{+}$ & & $1.20 \times 10^{-3}$ & $7.47 \times 10^{-3}$ & $2.78 \times 10^{-3}$ \\
\hline 3738 & 2307 & $5^{+}$ & & $2.70 \times 10^{-3}$ & $5.00 \times 10^{-3}$ & $6.72 \times 10^{-3}$ \\
\hline 3741 & 2310 & $4^{+}$ & $6.00 \times 10^{-4}$ & $4.80 \times 10^{-3}$ & $4.24 \times 10^{-3}$ & $4.16 \times 10^{-1}$ \\
\hline 3747 & 2316 & $5^{+}$ & & $1.28 \times 10^{-2}$ & $5.00 \times 10^{-3}$ & $3.26 \times 10^{-2}$ \\
\hline 3787 & 2356 & $2^{+}$ & $2.34 \times 10^{-2}$ & $2.00 \times 10^{-4}$ & $3.63 \times 10^{-3}$ & $1.82 \times 10^{1}$ \\
\hline 3793 & 2362 & $4^{+}$ & $2.00 \times 10^{-4}$ & $2.90 \times 10^{-2}$ & $2.20 \times 10^{-2}$ & $1.58 \times 10^{-1}$ \\
\hline 3883 & 2452 & $2^{+}$ & $7.40 \times 10^{-3}$ & $2.50 \times 10^{-3}$ & $8.77 \times 10^{-3}$ & 7.28 \\
\hline 3905 & 2474 & $1^{+}$ & $8.90 \times 10^{-3}$ & $3.60 \times 10^{-3}$ & $5.80 \times 10^{-3}$ & 9.22 \\
\hline 3905 & 2474 & $5^{+}$ & & $2.25 \times 10^{-2}$ & $5.00 \times 10^{-3}$ & $8.24 \times 10^{-2}$ \\
\hline 3962 & 2531 & $6^{+}$ & & $1.00 \times 10^{-4}$ & $2.11 \times 10^{-2}$ & $4.14 \times 10^{-4}$ \\
\hline 3977 & 2546 & $2^{+}$ & $1.56 \times 10^{-2}$ & $4.52 \times 10^{-2}$ & $2.55 \times 10^{-2}$ & $1.90 \times 10^{1}$ \\
\hline 3989 & 2558 & $6^{+}$ & & $5.30 \times 10^{-3}$ & $1.26 \times 10^{-2}$ & $2.32 \times 10^{-2}$ \\
\hline
\end{tabular}


TABLE II. The median and $1 \sigma$ upper and lower recommended rate as a function of temperature (GK).

\begin{tabular}{|c|c|c|c|}
\hline \multirow[b]{2}{*}{$T_{9}$} & \multicolumn{3}{|c|}{$N_{A}\langle\sigma \nu\rangle\left(\mathrm{cm}^{3} \mathrm{~mol}^{-1} \mathrm{~s}^{-1}\right)$} \\
\hline & $1 \sigma$ down & Median & $1 \sigma$ up \\
\hline 0.1 & $2.166 \times 10^{-19}$ & $2.177 \times 10^{-17}$ & $9.794 \times 10^{-17}$ \\
\hline 0.2 & $5.233 \times 10^{-12}$ & $1.903 \times 10^{-10}$ & $7.216 \times 10^{-10}$ \\
\hline 0.3 & $7.173 \times 10^{-9}$ & $1.836 \times 10^{-7}$ & $8.937 \times 10^{-7}$ \\
\hline 0.4 & $6.200 \times 10^{-7}$ & $6.576 \times 10^{-6}$ & $4.235 \times 10^{-5}$ \\
\hline 0.5 & $1.441 \times 10^{-5}$ & $6.487 \times 10^{-5}$ & $4.678 \times 10^{-4}$ \\
\hline 0.6 & $1.400 \times 10^{-4}$ & $3.414 \times 10^{-4}$ & $2.4971 \times 10^{-3}$ \\
\hline 0.7 & $7.347 \times 10^{-4}$ & $1.521 \times 10^{-3}$ & $9.193 \times 10^{-3}$ \\
\hline 0.8 & $2.633 \times 10^{-3}$ & $5.465 \times 10^{-3}$ & $2.389 \times 10^{-2}$ \\
\hline 0.9 & $7.566 \times 10^{-3}$ & $1.521 \times 10^{-2}$ & $4.926 \times 10^{-2}$ \\
\hline 1 & $1.760 \times 10^{-2}$ & $3.521 \times 10^{-2}$ & $9.631 \times 10^{-2}$ \\
\hline 1.5 & $3.048 \times 10^{-1}$ & $4.955 \times 10^{-1}$ & $8.588 \times 10^{-1}$ \\
\hline 2 & 1.424 & 2.023 & 2.972 \\
\hline 2.5 & 3.672 & 4.952 & 6.687 \\
\hline 3 & 7.324 & 9.247 & 11.6672 \\
\hline 3.5 & 13.089 & 15.5903 & 18.794 \\
\hline 4 & 22.597 & 25.4879 & 29.3052 \\
\hline 4.5 & 38.640 & 41.879 & 46.2883 \\
\hline 5 & 65.462 & 68.9195 & 73.7616 \\
\hline 6 & 174.35 & 178.033 & 183.316 \\
\hline 7 & 407.85 & 411.636 & 417.132 \\
\hline 8 & 842.41 & 846.079 & 851.543 \\
\hline 9 & 1567.96 & 1571.65 & 1577.11 \\
\hline 10 & 2684.66 & 2688.33 & 2693.03 \\
\hline
\end{tabular}

TABLE III. REACLIB fit coefficients for the recommended ${ }^{51} \mathrm{Fe}(\mathrm{p}, \gamma)$ reaction rate.

\begin{tabular}{cccc}
\hline \hline$a_{0}$ & $a_{1}$ & $a_{2}$ & $a_{3}$ \\
\hline $2.835035 \times 10^{2}$ & $-1.32948 \times 10^{1}$ & $5.385909 \times 10^{2}$ & $-8.624735 \times 10^{2}$ \\
$a_{4}$ & $a_{5}$ & $a_{6}$ & \\
\hline $5.228219 \times 10^{1}$ & -3.060584 & $4.138091 \times 10^{2}$ & \\
& & & \\
\hline \hline
\end{tabular}

